\title{
Genealogical concordance of phylogenetic species recognition-based delimitation of Neopestalotiopsis species associated with leaf spots and fruit canker disease affected guava plants
}

\author{
Imran Ul Haq ${ }^{1, *}$, Siddra $\mathrm{Ijaz}^{2}$ and Nabeeha Aslam Khan ${ }^{1}$ \\ ${ }^{1}$ Department of Plant Pathology University of Agriculture Faisalabad, Pakistan; ${ }^{2}$ Centre of Agricultural Biochemistry and \\ Biotechnology, University of Agriculture Faisalabad, Pakistan \\ *Corresponding author's e-mail: imran_1614@yahoo.com
}

\begin{abstract}
Neopestalotiopsis species are known to be involved in plant diseases as associated pathogens. The taxonomic identification of the fungal group Neopestalotiopsisis little bit difficult due to its complex evolutionary history. In the present study, seven fungal isolates were investigated from canker-affected guava plants. The phylogeny for generic placement of these isolates was analyzed to validate them as Neopestalotiopsis genus by phylogenetic signals from the 28S nrRNA region (LSU). Generated morphological data was segregated as new morpho-species of the Neopestalotiopsis genus. Hence, the internal transcribed spacer (ITS), Translation elongation factor 1- $\alpha$ (TEF1- $\alpha$ ) and Tubulin (TUB) genic region of these isolates were studied in juxtaposition with morphological data to resolve species limits. Both phylogenetic and morphological data revealed four novel species of the Neopestalotiopsis genus out of seven isolates studied. These Neopestalotiopsis species could be of great significance for further investigation as putative pathogens associated with canker or scabby canker disease in guava.

Keywords: Morphogenomics characterization, Operational taxonomy, neopestalotiopsis. novel species.
\end{abstract}

\section{INTRODUCTION}

Fungal plant pathogens, either true fungi or Oomycetes (fungal analogues), pose a significant threat to crops, animals and even human beings. Virulence behavior of any fungal species (even closely related) could be different in terms of their threat level (Rintoul et al., 2012). Thus, to specify fungi's species boundaries is indispensable for their effective management regarding disease control, phylogeographic distribution, and ecosystem conservation (Kohn, 2005). In Pakistan, most of the economically significant trees are attacked by different pathogens. Among these tree species, guava (Psidium guajava) is also severely affected by fungal pathogens (Amusa et al., 2005). Globally, guava production was recorded as fifty-five million tones; amongst guava producing countries, Pakistan (2.3 million tones) ranks at fifth production-wise, followed by India (21.8 million tones), China (4.8 million tones), Thailand (3.8 million tones), and Indonesia (3.1 million tones) (Guava production 2018). The decrease in guava's annual production was recorded from 509 to 495 thousand tons during 2015-2016, which was far less than the yield potential of this fruit tree. Diseases are the major factor, which contributes to yield loss. About one hundred and sixty-seven fungal pathogens reported have been associated with guava plant diseases (Misra, 2004). Besides, 21 fungi affecting the guava tree have been documented in Pakistan (Abbas et al., 2014).

There are different reports which support Neopestalotiopsis species as pathogenic to the kingdom, Plantae (Hyde et al., 2014; Jayawardene et al., 2016) and cause severe losses to various economically important plant species (Maharachchikumbura et al., 2013). In this study, different isolates of Neopestalotiopsis species associated with guava plants were identified. These investigated fungal isolates were collected by the research team of "Fungal Molecular Biology (FMB) Laboratory" Department of Plant Pathology, University of Agriculture Faisalabad, Pakistan while surveying to document data regarding the prevalence of fungal diseases of $P$. guajava and to detect the association of fungal pathogens with disease affected plants. These fungal isolates were characterized on a morphological basis through microscopy and on a molecular level by using molecular biology and computational biology approaches. Investigation of these fungal isolates on a morphogenomics basis supported operational taxonomy for species identification. Because fungi were identified as morphospecies generally, however, identification on the morphological basis is not considered an authenticated way to define species boundaries until or unless

Haq Ul I., S. Ijaz. N. A. khan. 2021. Genealogical concordance of phylogenetic species recognition-based delimitation of Neopestalotiopsis species associated with leaf spots and fruit canker disease affected guava plants. Pak. J. Agri. Sci. 58:1301-1313.

[Received 28 Aug 2020; Accepted 18 Jun 2021; Published (online) 21 Sep 2021] 
supported by molecular phylogenetic separation (Taylor et al., 2000). A single morphospecies with broad-based distribution may be complex cryptic species, which could be different unidentified phylogenetic species (Dettman et al., 2003). Nowadays, operational taxonomy mainly relies on DNA sequences of multiple loci for resolving cryptic species that had been considered single morphospecies previously (Denman et al., 2000). The phylogenetic species recognition approach identified new sibling species within well described (on morphology) taxa (Pavlic et al., 2007). Phylogenetic species recognition strategy is based on single-locus analysis concatenated analysis using multiple loci and coalescent theory-based analysis. A significant reproach has been facing single locus phylogenetic analysis for species identification and species delimitation, as it may not represent an individual as a whole (Smith and Todd, 2001). Therefore, in this study, morphological identification of fungal isolates (FMB isolates) was supplemented with phylogenetic analyses to delimit and specify their species boundaries. The polyphasic approach was used to achieve the goal, including morphometric evaluation, single-gene genealogies estimation, multiple locibased analyses (concatenated analyses), and a coalescent theory-based method species delineation.

\section{MATERIALS AND METHODS}

Sampling, isolation and initial categorization: The Fungal Molecular Biology (FMB) Laboratory carried out surveys of Pakistan's guava-growing regions during 2016 and 2017 (Table 1). Ninety guava orchards covering 24 districts across the country were visited. The detection of fungal pathogens associated with canker or scabby canker affected guava plant samples comprising fruits, leaves, twigs, and branches. Evaluations were conducted by investigating approx. Two hundred fruits, twigs portions and living leaves samples are collected each year. Before cutting the samples into small pieces $(\sim 0.5 \mathrm{~cm})$, samples (Canker/ Scabby canker affected branches, leaves, fruits) were thoroughly washed with tap water to remove dirt. Surface sterilization was done by oneminute dipping into $2 \%$ sodium hypochlorite solution followed by washings with sterilized distilled water. Samples were placed on blotter paper to remove excess water from these bits before transferring them on the Petri plates containing Potato Dextrose Agar (PDA) medium.

Morphological Characterization: Initially, sixty-three isolates of Neopestalotiopsis were obtained and multiplied on PDA at $25^{\circ} \mathrm{C} \pm 2$ under continuous illumination. Firstly, Neopestalotiopsis isolates were identified microscopically using classical taxonomic criteria described by Guba (1961), Sutton (1980) and Maharachchikumbura et al. (2013). The measurements of relevant morphological characters were made from 30 arbitrarily selected conidia from a conidial suspension of each isolate prepared in sterile distilled water using compound microscope Meji Techno; Japan model HD1600T fitted with Olympus (DP25) digital camera. Seven distinct Neopestalotiopsis isolates were identified, having variation in their morphological characters among all the examined isolates. All seven distinct isolates were further subjected to phylogenetic analyses for validated identification.

DNA extraction, PCR analysis and DNA sequencing: DNA from fresh mycelia of studied fungal isolates was extracted using GeneJET Genomic DNA purification kit (Thermo scientific). For Phylogenetic analyses of investigated fungal isolates, four taxonomically informative loci, LSU, ITS, TEF1- $\alpha$ and tubulin, were examined. PCR analysis was performed using gene-specific primers of each investigated loci in Veriti TM 96 wells thermal cycler of Applied Biosystems. PCR conditions for amplifying these genic regions were optimized through gradient PCR comprised of various thermal and reagent profiles. Partial regions of these genes were amplified using primer pair ITS1-F/ITS4, LROR/LR7, EF-728/EF-2 and TI/Bt2b (Table 2).

PCR products were resolved on $1 \%$ agarose gel and were visualized under UV light in Gel documentation system (Bio$\mathrm{Rad}$ ). Amplicons (of required size) of each genetic region were purified using GeneJET PCR purification kit (Thermo Scientific) and were sequenced through Eurofins Genomics DNA sequencing services. Sequences were trimmed using sequence alignment editor, BioEdit version 7.2.6.1. Partial sequences of these genic regions were generated using both forward and reverse primers and their consensus sequences were obtained using DNASTAR Lasergene v. 7.1.0 SeqMan pro (SeqManTMII). All generated DNA sequences were deposited in GenBank (Table 3).

Phylogenetic analyses: For alignment and phylogenetic comparison, an individual dataset of each gene and concatenated dataset of all genes were made. For this, generated sequences studied were supplemented with available sequences from GenBank based on homology and literature. Generated DNA sequences were queried against the database by subjecting them to homology search tool BLAST (Basic Local Alignment Search Tool) and sequences from GenBank based on similarity to which queried sequences got maximum similarities were obtained (Table 4). A partition homogeneity (ILD) test was conducted using PAUP* version 4.0a161 to evaluate statistical congruence, determining datasets' combinability. This test was performed in the heuristic standard search option to check whether individual data partitions could be used as a combined dataset for phylogenetic analyses. The concatenated dataset from sequence data of individual partitions was generated with Geneious software v. 4.8.5. Then single nexus file of the concatenated dataset was generated using Mesquite version 3.51. Multiple sequence alignment was constructed using multiple sequence alignments (MSA) tools, such as MUSCLE and ClustalW. For getting more accuracy and reliability of MSA-based phylogenomic analyses, aligned sequence datasets were trimmed with Gblocks server (Castresana, 2000 ) to eliminate poorly aligned regions from sequence data. For a preliminary assessment of phylogenetically diverse clades, Neighbor-joining (NJ) analysis for phylogenetic tree construction was executed on a concatenated dataset of all genes used in this study MEGA6.06 software. 
Table 1. Areas surveyed covering 90 Guava orchards, 24 districts across the country for isolation of fungi associated with Psidium guajava.

\begin{tabular}{|c|c|c|c|c|}
\hline \multirow{2}{*}{\multicolumn{5}{|c|}{$\begin{array}{cc}\text { Districts } & \text { Location } \\
\text { PUNJAB PROVINCE PAKISTAN }\end{array}$}} \\
\hline & & & & \\
\hline \multirow[t]{5}{*}{1.} & District Faisalabad & UAF & $31.4303,73.0672$ & Psidium guajava \\
\hline & & PARS & $31.3630,72.9876$ & Psidium guajava \\
\hline & & Samundri & $31.0646,72.9520$ & Psidium guajava \\
\hline & & Jaranwala & $31.3454,73.4298$ & Psidium guajava \\
\hline & & Tandlianwala & $31.0368,73.1379$ & Psidium guajava \\
\hline \multirow[t]{2}{*}{2.} & District Bahawalpur & Bahawalpur & $29.3544,71.6911$ & Psidium guajava \\
\hline & & Lal Suhanra & $29^{\circ} 19,71^{\circ} 55$ & Psidium guajava \\
\hline \multirow[t]{5}{*}{3.} & District Bahawalnagar & Bahawalnagar & $30.0025,73.2412$ & Psidium guajava \\
\hline & & Fort Abbas & $29.1931,72.8575$ & Psidium guajava \\
\hline & & Dolat pur & $32^{\circ} 41,73^{\circ} 18$ & Psidium guajava \\
\hline & & Chishtian & $29.7956,72.8634$ & Psidium guajava \\
\hline & & Haroonabad & $29.6081,73.1468$ & Psidium guajava \\
\hline \multirow[t]{2}{*}{4.} & District Layyah & Layyah & $30.9693,70.9428$ & Psidium guajava \\
\hline & & Chowk Azam & $30.9647,71.2043$ & Psidium guajava \\
\hline 5. & District Chiniot & Chiniot & $31.7292,72.9822$ & Psidium guajava \\
\hline 6. & District Hafizabad & Hafizabad & $32.0712,73.6895$ & Psidium guajava \\
\hline 7. & District Kasur & Pattoki & $31.0249,73.8479$ & Psidium guajava \\
\hline 8. & District Nankana & Shah kot & $31.5757,73.4815$ & Psidium guajava \\
\hline \multirow[t]{2}{*}{9.} & District Sheikhupura & Sheikhupura & $31.7167,73.9850$ & Psidium guajava \\
\hline & & Sharak Pur & $31^{\circ} 27^{\prime} 28.8,74^{\circ} 06^{\prime} 00.0$ & Psidium guajava \\
\hline 10. & District Multan & Multan & $30.1575,71.5249$ & Psidium guajava \\
\hline 11. & District Vehari & Burewala & $30.1593,72.6943$ & Psidium guajava \\
\hline 12. & District Sargodha & Sargodha & $32.0740,72.6861$ & Psidium guajava \\
\hline \multicolumn{5}{|c|}{ SINDH PROVINCE PAKISTAN } \\
\hline 1. & District Banbhore & Thatta & $24.7475,67.9106$ & Psidium guajava \\
\hline \multirow[t]{4}{*}{2.} & District Hyderabad & Hyderabad & $25.3960,68.3578$ & Psidium guajava \\
\hline & & Jamshoro & $25.4304,68.2809$ & Psidium guajava \\
\hline & & Dadu & $26.7341,67.7795$ & Psidium guajava \\
\hline & & Tando Jahanian & $25.3924,68.3507$ & Psidium guajava \\
\hline 3. & $\begin{array}{l}\text { District Shaheed } \\
\text { Benazir Abad }\end{array}$ & Naushahro Feroze & $26.8463,68.1253$ & Psidium guajava \\
\hline 4. & District Larkana & Larkana & $27.5570,68.2028$ & Psidium guajava \\
\hline \multicolumn{5}{|c|}{ KPK PROVINCE PAKISTAN } \\
\hline 1. & District Bannu & Bannu & $32.9910,70.6455$ & Psidium guajava \\
\hline \multirow[t]{2}{*}{2.} & District Hazara & Hazara & $32.7962,74.2840$ & Psidium guajava \\
\hline & & Haripur & $33.9946,72.9106$ & Psidium guajava \\
\hline 3. & District Mardan & Mardan & $34.1989,72.0231$ & Psidium guajava \\
\hline 4. & District Kohat & Kohat & $33.5889,71.4429$ & Psidium guajava \\
\hline \multicolumn{5}{|c|}{ BLOCHISTAN PROVINCE PAKISTAN } \\
\hline 1. & District Kalat & Lasbela & $29.0523,66.5879$ & Psidium guajava \\
\hline 2. & District Makran & Gwadar & $25.2460,62.2861$ & Psidium guajava \\
\hline 3. & District Nasirabad & Jafarabad & $28.0063,67.8430$ & Psidium guajava \\
\hline 4. & District Sibi & Sibi & $29.5532,67.8808$ & Psidium guajava \\
\hline
\end{tabular}

Table 2. Primers of different loci (ITS, LSU, TEF1- $\alpha$, and tubulin) used for phylogenetic analyses

\begin{tabular}{lll}
\hline Sr\# & Primer pair & References \\
\hline 1 & ITS1-F/ITS4 & White et al., 1990 \\
2 & LROR/LR7 & Vilgalys and Hester 1990 \\
3 & EF-728/EF-2 & Carbone and Kohn, 1999; O' Donnell et al., 198 \\
4 & TI/Bt2b & O'Donnell and Cigelnik 1996; Glass and Donaldson, 1995 \\
\hline
\end{tabular}


Table 3. GenBank accession numbers assigned to FMB fungal isolates of Guava tree by NCBI database

\begin{tabular}{|c|c|c|c|c|c|c|}
\hline \multirow[t]{2}{*}{ Sr\# } & \multirow[t]{2}{*}{ Isolate } & \multirow[t]{2}{*}{ Substrate } & \multicolumn{4}{|c|}{ GenBank accession numbers } \\
\hline & & & ITS & TEF1- $\alpha$ & TUB & LSU \\
\hline 1 & FMB-Guv-B3 & Branches & MF783085 & MH460868 & MH460871 & MH478111 \\
\hline 2 & FMB-Guv-C4 & Branches & MF783084 & MH460869 & MH460872 & MH478106 \\
\hline 3 & FMB-Guv-MB & Branches & MF783082 & MH460874 & MH477870 & MH478110 \\
\hline 4 & FMB-Guv-JF2 & Fruit & MH209077 & MH523647 & МH460876 & MH478107 \\
\hline 5 & FMB-Guv-JF7 & Fruit & MH209246 & MH523646 & MH460875 & MH478112 \\
\hline 6 & FMB-Guv-L2A & Leaves & MH209245 & MH460870 & MH460873 & MH478108 \\
\hline 7 & FMB-Guv-B2A & Branches & MH208973 & MH523648 & MH477871 & MH478109 \\
\hline
\end{tabular}

Table 4. List of Neopestalotiopsis and Pestalotiopsis with associated host, used for constructing phylograms.

\begin{tabular}{|c|c|c|c|}
\hline Species/Isolate & Culture Accession \# & Host & Country \\
\hline FMB-Guv-B3 & FMB0026 & Guava & Pakistan \\
\hline FMB-Guv-C4 & FMB0027 & Guava & Pakistan \\
\hline FMB-Guv-MB & FMB0028 & Guava & Pakistan \\
\hline FMB-Guv-JF2 & FMB0127 & Guava & Pakistan \\
\hline FMB-Guv-JF7 & FMB0128 & Guava & Pakistan \\
\hline FMB-Guv-L2A & FMB0129 & Guava & Pakistan \\
\hline FMB-Guv-B2A & FMB0130 & Guava & Pakistan \\
\hline Neopestalotiopsis aotearoa & CBS-367.54 & Canvas & New Zealand \\
\hline Neopestalotiopsis asiatica & MFLUCC12-0286 & - & China \\
\hline Neopestalotiopsis australis & CBS-114159 & Telopea & Australia \\
\hline Neopestalotiopsis chrysea & MFLUCC12-0261 & - & China \\
\hline Neopestalotiopsis clavispora & CBS-447.73 & - & Sri Lanka \\
\hline Neopestalotiopsis cubana & CBS-600.96 & - & Cuba \\
\hline Neopestalotiopsis ellipsospora & CBS-115113 & Coralberry & China \\
\hline Neopestalotiopsis eucalypticola & CBS-264.37 & Eucalyptus & - \\
\hline Neopestalotiopsis formicarum & CBS-115.83 & - & Cuba \\
\hline Neopestalotiopsis honoluluana & CBS-111535 & Telopea & USA \\
\hline Neopestalotiopsis honoluluana & CBS 114495 & Telopea & USA \\
\hline Neopestalotiopsis javaensis & CBS 257.31 & Coconut & Indonesia \\
\hline Neopestalotiopsis mesopotamica & CBS 299.74 & Eucalyptus & Turkey \\
\hline Neopestalotiopsis mesopotamica & CBS 336.86 & Calabrian Pine & Iraq \\
\hline Neopestalotiopsis natalensis & CBS 138.41 & Black Wattle & South Africa \\
\hline Neopestalotiopsis piceana & CBS 225.30 & Dica & \\
\hline Neopestalotiopsis piceana & CBS 254.32 & Coconut & Indonesia \\
\hline Neopestalotiopsis piceana & CBS 394.48 & Spruce & United Kingdom \\
\hline Neopestalotiopsis protearum & CBS 114178 & Wart-stemmed pincushion & Zimbabwe \\
\hline Neopestalotiopsis rosae & CBS 101057 & Rose & New Zealand \\
\hline Neopestalotiopsis rosae & CBS 124745 & Moutan peony & USA \\
\hline Neopestalotiopsis samarangensis & CBS 115451 & - & China \\
\hline Neopestalotiopsis saprophytica & CBS 115452 & Oblong leaved litsea & China \\
\hline Neopestalotiopsis surinamensis & CBS 450.74 & African oil palm & Suriname \\
\hline Neopestalotiopsis foedans & CGMCC 3.9123 & Mangrove & China \\
\hline Neopestalotiopsis foedans & CGMCC 3.9178 & Triangle palm & China \\
\hline Neopestalotiopsis musae & MFLUCC 15-0776 & Musa & Thailand \\
\hline Neopestalotiopsis saprophytica & MFLUCC 12-0282 & - & China \\
\hline Pestalotiopsis malayana & CBS 102220 & Mahang & Malaysia \\
\hline Pestalotiopsis colombiensis & CBS 118553 & Eucalyptus & Colombia \\
\hline Pestalotiopsis monochaeta & CBS 144.97 & Oak & Netherlands \\
\hline Pestalotiopsis arceuthobii & CBS 434.65 & western dwarf mistletoe & USA \\
\hline Pestalotiopsis arengae & CBS 331.92 & Batbat palm & Singapore \\
\hline Pestalotiopsis australasiae & CBS 114141 & Protea & Australia \\
\hline
\end{tabular}


GCPSR based delimitation of Neopestalotiopsis Species

\begin{tabular}{|c|c|c|c|}
\hline Species/Isolate & Culture Accession \# & Host & Country \\
\hline Pestalotiopsis australis & CBS 114193 & Grevillea & Australia \\
\hline Pestalotiopsis australis & CBS 119350 & Ghoeboontjie & South Africa \\
\hline Pestalotiopsis biciliata & CBS 124463 & London plane & Slovakia \\
\hline Pestalotiopsis biciliata & CBS 790.68 & conifer & Netherlands \\
\hline Pestalotiopsis camelliae & CBS 443.62 & - & - \\
\hline Pestalotiopsis camelliae & MFLUCC 12-0278 & Common camellia & China \\
\hline Pestalotiopsis chamaeropis & CBS 113604 & - & - \\
\hline Pestalotiopsis chamaeropis & CBS 186.71 & European fan palm & Italy \\
\hline Pestalotiopsis chamaeropis & CBS 237.38 & - & Italy \\
\hline Pestalotiopsis diploclisiae & CBS 115585 & Diploclisia & China \\
\hline Pestalotiopsis diploclisiae & CBS 115587 & Diploclisia & China \\
\hline Pestalotiopsis furcata & MFLUCC 12-0054 & - & Thailand \\
\hline Pestalotiopsis grevilleae & CBS 114127 & Grevillea & Australia \\
\hline Pestalotiopsis hawaiiensis & CBS 114491 & Leucospermum & USA \\
\hline Pestalotiopsis hollandica & CBS 265.33 & Japanese umbrella pine & Netherlands \\
\hline Pestalotiopsis humus & CBS 336.97 & - & Papua New Guinea \\
\hline Pestalotiopsis jesteri & CBS 109350 & Fagraea tree & Papua New Guinea \\
\hline Pestalotiopsis kenyana & CBS 911.96 & - & - \\
\hline Pestalotiopsis knightiae & CBS 111963 & Knightia & New Zealand \\
\hline Pestalotiopsis novae-hollandiae & CBS 130973 & Bull Banksia & Australia \\
\hline Pestalotiopsis oryzae & CBS 171.26 & - & Italy \\
\hline Pestalotiopsis papuana & CBS 331.96 & - & Papua New Guinea \\
\hline Pestalotiopsis parva & CBS 265.37 & Flamboyant & - \\
\hline Pestalotiopsis parva & CBS 278.35 & Drooping laurel & - \\
\hline Pestalotiopsis portugalica & CBS 393.48 & - & Portugal \\
\hline Pestalotiopsis scoparia & CBS 176.25 & Conifers & - \\
\hline Pestalotiopsis spathulata & CBS 356.86 & Chilean hazelnut & Chile \\
\hline Pestalotiopsis telopeae & CBS 113606 & Telopea & Australia \\
\hline Pestalotiopsis telopeae & CBS 114137 & Protea & Australia \\
\hline Pseudopestalotiopsis cocos & CBS 272.29 & Coconut & Indonesia \\
\hline Pseudopestalotiopsis theae & MFLUCC 12-0055 & Tea plant & Thailand \\
\hline
\end{tabular}

FMB: Fungal Molecular Biology Laboratory Culture Collection, University of Agriculture Faisalabad, Pakistan; CBS: Culture Collection of the Centraalbureau voor Schimmelcultures, Fungal Biodiversity Centre, Utrecht, The Netherlands; MFLUCC: Mae Fah Luang University Culture Collection, Thailand; JZB Culture Collection of Beijing Academy of Agriculture and Forestry Sciences, Beijing, China; CGMCC: China General Microbiological Culture Collection Center, Institute of Microbiology, Chinese Academy of Sciences, Beijing, China.

For phylogenetic analyses of individual gene datasets and combined datasets of all genes, Maximum parsimony (MP) analysis and Bayesian inference (BI) were employed for tree construction. PAUP* v. 4.0a161 software was used for Maximum parsimony analysis, whereas MrBayes v. 3.2.6 was applied for Bayesian inference (BI) and the most fit model for Bayesian analysis was selected by MrModeltest v.2.3. For individual data partitions and concatenated datasets of three loci (ITS, TEF1- $\alpha$, TUB), maximum-parsimony genealogies were generated using the Bootstrap method in full heuristic search with 1000 bootstrap replicates and 10 replicates of random stepwise addition. Tree-bisection-reconstruction (TBR) was selected as a branch swapping algorithm with a reconnection limit of, 8 and branches were collapsed when the maximum branch length was zero. Gaps were treated as a new state (5th base), 'MulTrees' option was in effect. All characters were of unord type and had equal weight.
For parsimony analysis, different metrics included tree length (TL), homoplasmy index, consistency index (CI), rescaled consistency index (RC), and retention index (RI), were calculated. Bayesian inference (BI) analysis was executed on a concatenated dataset for genealogical concordance of phylogenetic species recognition (GCPSR). The most suitable evolutionary model for the Bayesian approach was selected for a concatenated dataset of all three genes. MrModeltest applying Akaike information criterion (AIC) determined nucleotide substitution model. Four simultaneous Markov Chain Monte Carlo (MCMC) chains were run for 5,000,000 generations with 0.25 burnin and 1000 sample frequency. This MCMC run was stopped as the average value of split frequencies reached below 0.01. The output files (MCMC trace files) generated through Bayesian were analyzed with Tracer v.1.5, and a phylogenetic tree was visualized using FigTree version 1.4.3. To validate concatenated analysis for 
species delimitation, the coalescent species tree method was used to construct species tree by implementing *BEAST software v. 2.5.0. Alignment files in NEX format were imported to BEAUti (Bayesian Evolutionary Analysis Utility) program under a non-standard template, StarBEAST, to set up the evolutionary model and MCMC run to generate the XML file. This XML was used as an input file for BEAST (Bayesian Evolutionary Analysis for Sampling Tree). Linear and constant root models were used as multi-species coalescent model. Yule model was implemented as species tree priors to specify speciation time distribution. The maximum clade credibility species tree was generated by TreeAnnotator version 2.3 .0 with $10 \%$ burin and a 0.5 posterior probability limit to identify a tree, representing the best posterior distribution. The species tree was visualized in Figtree software.

Preservation of fungal isolates: Single spore cultures of seven Neopestalotiopsis isolates were derived and preserved using agar slants at $4^{\circ} \mathrm{C}$ and dry filter papers (Fong et al., 2000). All isolates were deposited in Fungal Molecular Biology Laboratory Culture Collection (FMB-CC) the University of Agriculture Faisalabad, having World Data Center for Microorganism (WDCM) Registration ID 1155, an affiliate member of World Federation for Culture Collection having Global Catalogue for Microorganism (GCM) web page http://gcm.wfcc.info/cc/fmb-cc-uaf.

\section{RESULTS}

\section{TAXONOMY}

Neopestalotiopsis guajavae I.U Haq, S. Ijaz \& N. A. Khan, sp.nov. MycoBank 840596

Basionym: Neopestalotiopsis Maharachch., K.D. Hyde \& Crous, Studies in Mycology 79: 135 (2014) [MB\#809759]

Material examined: Pakistan, Punjab, from living branches of Guava tree, 11 Apr. 2017, I.U Haq (FMB H 11.1, Holotype, ex-type culture FMBCC 11.1= FMB0026-FMB0027)

Note: The strain is preserved in a metabolically inactive state. Etymology: Named after the host (Guava, in English; Peruka, in Sanskrit; Psidium guajava, botanical name) from which these were isolated.

Colonies after seven days of inoculation at $27-28,{ }^{\circ} \mathrm{C}$ with white-colored concentric aerial mycelium and black conidiomata with undulating edge appeared on the PDA medium. The conidia were straight to slightly curved with four septations, discrete cells having $23.1 \pm 1.4 \mu \mathrm{m}$ length and $7.2 \pm 0.4 \mu \mathrm{m}$ width. Conidia produced versicolourous three median cells. The first two median cells were brown, having $4.8 \pm 0.4 \mu \mathrm{m}$ and $5.3 \pm 0.4 \mu \mathrm{m}$ length respectively, while the third cell was light yellow with $5.1 \pm 0.3 \mu \mathrm{m}$ length. Septa were darker in colour than the rest of the cells. The apical cell was $\mathrm{V}$-shaped, hyaline smooth and thin-walled, having a length of $3.9 \pm 0.7 \mu \mathrm{m}$. One to two tubular apical appendages $20.8 \pm 4.1$ $\mu \mathrm{m}$ long were raised from the top of the apical cell. The conical basal cell was $3.7 \pm 0.6 \mu \mathrm{m}$ long with a thin rugose wall. The basal appendage was filiform, straight to slightly curved, $5.7 \pm 1.2 \mu \mathrm{m}$ long.

Neopestalotiopsis psidii I.U Haq, S. Ijaz \& N. A. Khan, sp.nov. MycoBank MB840638

Basionym: Neopestalotiopsis Maharachch., K.D. Hyde \& Crous, Studies in Mycology 79: 135 (2014) [MB\#809759]

Material examined: Pakistan, Punjab, from living branches of Guava tree, 11 Apr. 2017, I.U Haq (FMB H 11.2, Holotype, ex-type culture FMBCC 11.2= FMB0028)

Note: The strain is preserved in a metabolically inactive state.

Etymology: Named after the host (Guava, in English; Peruka, in Sanskrit; Psidium guajava, botanical name) from which these were isolated.

The Colonies on the PDA medium after seven days at 27$28^{\circ} \mathrm{C}$ appeared with white to grey moderate aerial mycelium, lobate edge, black colored gregarious conidiomata on mycelium's surface. Conidia were straight to slightly curved, fusoid with four septations having $24 \pm 1.0 \mu \mathrm{m}$ length and $6.6 \pm 0.7 \mu \mathrm{m}$ width, having four septations with three versicolourous median cells. The first two median cells were honey yellow colored having length of $5 \pm 0.5 \mu \mathrm{m}$ and $5.3 \pm 0.6$ $\mu \mathrm{m}$, respectively, while the third median cell was light yellow and was $4.8 \pm 0.6 \mu \mathrm{m}$ long. Septa were darker than the rest of the cells. The apical cell was hyaline and cylindrical, having a length of $4.3 \pm 0.6 \mu \mathrm{m}$. Two tubular apical appendages of length $20.5 \pm 5.6 \mu \mathrm{m}$ length were raised from the top of the apical cell. The basal cell was obconic to conical, having $4.4 \pm 0.4 \mu \mathrm{m}$ length. Straight to curved basal appendage was produced with $4 \pm 1.5 \mu \mathrm{m}$ length.

Neopestalotiopsis perukae I.U Haq, S. Ijaz \& N. A. Khan, sp.nov. MycoBank MB840637

Basionym: Neopestalotiopsis Maharachch., K.D. Hyde \& Crous, Studies in Mycology 79: 135 (2014) [MB\#809759]

Material examined: Pakistan, Punjab, from living branches of Guava tree, 11 Apr. 2017, I.U Haq (FMB H 11.3, Holotype, ex-type culture FMBCC 11.3= FMB0127- FMB0128- FMB0130)

Note: The strain is preserved in a metabolically inactive state.

Etymology: Named after the host (Guava, in English; Peruka, in Sanskrit; Psidium guajava, botanical name) from which these were isolated. 
Colonies on the PDA medium after seven days at $27-28^{\circ} \mathrm{C}$ were whitish to pale white dense aerial mycelium with the smooth edge with yellowish gregarious conidiomata immersed in the mycelium. Conidia were straight to slightly curved, fusiform, ellipsoid having length $19.7 \pm 1.4 \mu \mathrm{m}$ and width $6.4 \pm 0.8 \mu \mathrm{m}$. Conidia were produced with four septations and three versicolourous median cells. The upper two median cells were dark brown to black, with $4.35 \pm 0.4 \mu \mathrm{m}$ and $4.4 \pm 0.4 \mu \mathrm{m}$ length respectively, the third median cell was pale yellow and $4.6 \pm 0.6 \mu \mathrm{m}$ long. The wall between the upper two median cells was relatively thicker and darker, sub cylindrical hyaline thin-walled apical cell with $3.6 \pm 0.7 \mu \mathrm{m}$ length. Two unbranched, un-knobbed tubular apical appendages attached to the middle portion of the apical cell were $22.2 \pm 5.8 \mu \mathrm{m}$ long. The hyaline obconic basal cell was $3.1 \pm 0.5 \mu \mathrm{m}$ long, with a posterior appendage of $3.8 \pm 1.9 \mu \mathrm{m}$ length.

Neopestalotiopsis guajavicola I.U Haq, S. Ijaz \& N. A. Khan, sp.nov. MycoBank MB840639

Basionym: Neopestalotiopsis Maharachch., K.D. Hyde \& Crous, Studies in Mycology 79: 135 (2014) [MB\#809759]

Material examined: Pakistan, Punjab, from living branches of Guava tree, 11 Apr. 2017, I.U Haq (FMB H 11.4, Holotype, ex-type culture FMBCC 11.4= FMB0129)

Note: The strain is preserved in a metabolically inactive state.

Etymology: Named after the host (Guava, in English; Peruka, in Sanskrit; Psidium guajava, botanical name) from which these were isolated.

Colonies of isolate FMB-Guv-L2A produced undulating edge white to honey-coloured moderate aerial mycelium with black-colored gregarious conidiometa on the PDA medium after seven days at $27-28^{\circ} \mathrm{C}$. The Conidia were straight, relatively broader, ellipsoid, clavate having length $23.3 \pm 1.6$ $\mu \mathrm{m}$ and width $6.5 \pm 0.5 \mu \mathrm{m}$. The conidia were containing four Septations and three median cells. The first two median cells were yellow to brown, having $4.8 \pm 0.3 \mu \mathrm{m}$ and $5.1 \pm 0.2 \mu \mathrm{m}$ length respectively, the third cell was light yellow with $4.8 \pm 0.5 \mu \mathrm{m}$ length. Septa were darker than the rest of the cell. The apical cell was relatively small, hyaline having $4.4 \pm 0.4$ $\mu \mathrm{m}$ length with a very thin wall. Two to three tubular unbranched apical appendages having 21.8 $\pm 2.7 \mu \mathrm{m}$ length were produced. In addition, a conical basal cell $4.0 \pm 0.5 \mu \mathrm{m}$ long with a single unbranched basal appendage $4.4 \pm 1.5 \mu \mathrm{m}$ in length was observed.

\section{OPERATIONAL TAXONOMY}

PCR analysis and sequencing: Seven fungal isolates from guava canker-affected plants were investigated through phylogenetic reconstructions based on different phylogenomics methods. Extracted DNA was quantified and a dilution of $50 \mathrm{ng} / \mu \mathrm{l}$ was made for each fungal isolates. PCR analysis products with sequence-specific primer pair of each locus (ITS, TEF1- $\alpha$, TUB and LSU) were resolved using agarose gel to check amplicon sizes. PCR products were purified with a GeneJET PCR purification kit (Thermo Scientific) and then were sequenced. Sequences generated were lodged in GenBank with accession numbers described in Table 3. For phylogenetic analyses, generated sequences were aligned with sequences obtained from the NCBI database after BLAST these sequences and with literature support as well, given in Table 4. Alignments were created with the MSA tool, ClustalW and were trimmed with the Gblocks server.

Phylogenetic analyses: As literature documented, the generic placement of members belongs to Pestalotiopsis and Neopestalotiopsis genera have been controversial. Therefore, for validating our morphometric characterization of studied isolates as Neopestalotiopsis, phylogenetic analysis by aligning LSU sequences generated in this study with sequences of Pestalotiopsis, Pseudopestalotiopsis and Neopestalotiopsis from the NCBI database was made. So, a neighbor-joining tree was constructed through the MEGA6.06 software package. For neighbor-joining analysis, a Bootstrap method with 1000 replications was used. This phylogenetic tree (Fig. 1) gave a good resolution for placing fungal isolates (studied here) at the generic level. This tree showed all the isolates made cladding with the Neopestalotiopsis group, as shown in Figure 1. These fungal isolates were delimited by clarifying their species boundaries by analyzing a single locus dataset and a combined dataset of three genes (ITS, TEF1- $\alpha$, TUB). For genealogical concordance of phylogenetic species recognition (GCPSR), LSU was excluded from concatenated analyses and coalescent species tree delimitation methods. Exclusion of LSU dataset from further analysis for species delimitation of these isolates based on its parsimony-informative character (PIC) was one. Phylogenies from each genetic region dataset and combined dataset of all three genes were determined using PAUP* v. 4.0a161software. For the concatenated dataset of genes (ITS, TEF1- $\alpha$, TUB), the Bayesian inference (BI) method was also applied. MrModeltest v.2.3 was used to predict the evolutionary model for BI analysis, with the proportion of invariable sites. Phylogenetic trees generated from maximum parsimony analysis and Bayesian inference were shown similar tree topologies. Gene genealogies from the dataset of each gene were similar to concatenated dataset phylogenies.

Phylogenetic analysis for estimating species boundaries: The maximum parsimony method was used first to obtain genealogies from four single loci (ITS, TEF1- $\alpha$, TUB and LSU) alignments separately. Phylogenetic reconstruction of all trees was shown a similar pattern with slight differences. 


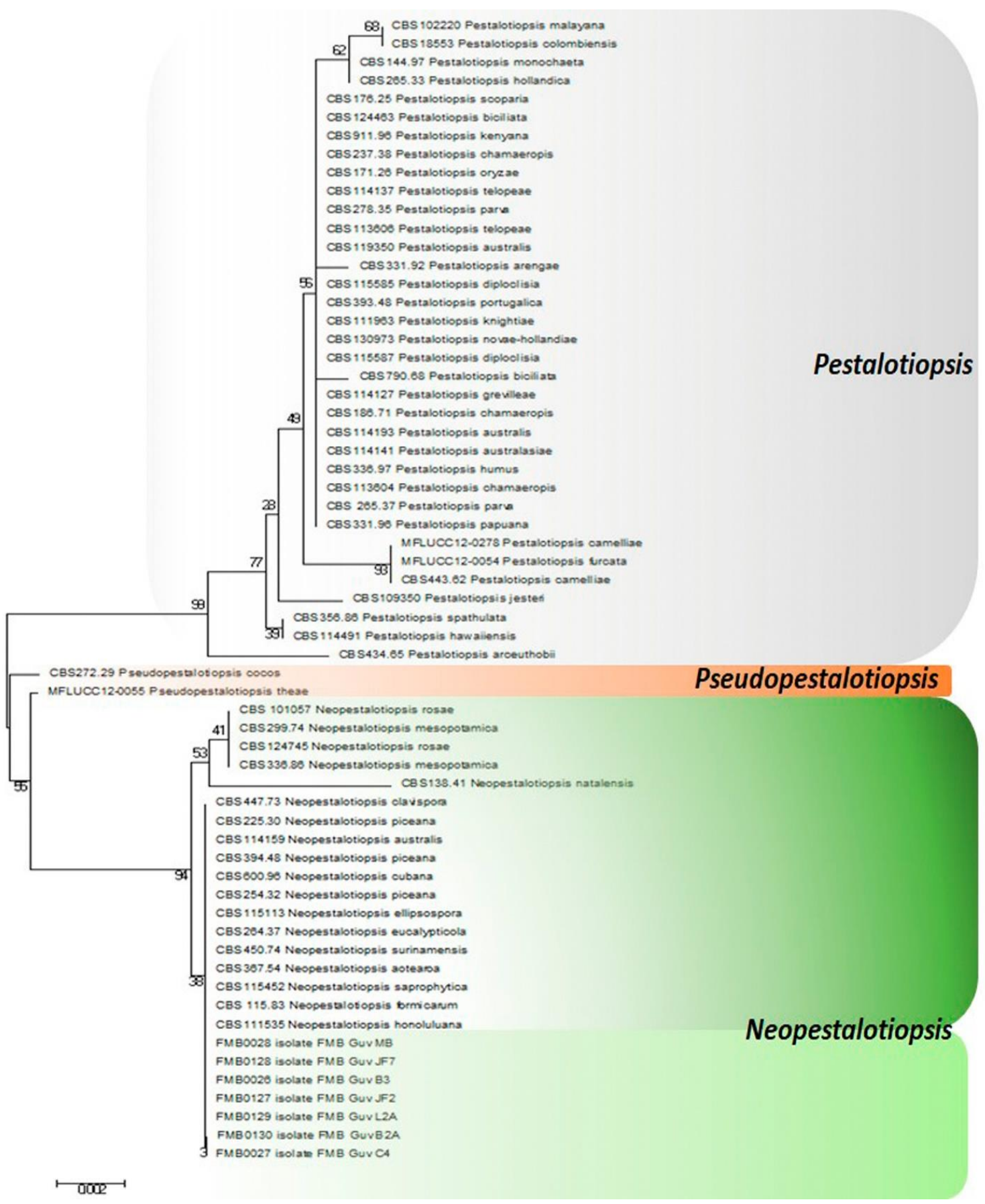

Figure 1. Phylogram resulting from Neighbor-joining analysis of LSU sequence alignment of Pestalotiopsis, Neopestalotiopsis, and Pseudopestalotiopsis. These genera are represented with colored blocks. This phylogenetic tree is providing clear-cut resolution to FMB isolates with their respective genus, Neopestalotiopsis. Scale bar is depicting the expected number of changes per site.

The maximum parsimony tree of the TEF1- $\alpha$ region was based on 28 parsimony-informative characters (PIC), with tree length 110 . The calculated matrices of this analysis were 0.2182 homoplasmy index (HI), 0.7818 consistency index
(CI), 0.5018 rescaled consistency index (RC) and 0.6418 retention index (RI). However, the Tubulin (TUB) region's maximum parsimony tree was based on 456 parsimonyinformative characters (PIC), with tree length 693. 
The calculated matrices of this analysis were 0.0779 homoplasmy index (HI), 0.9221 consistency index (CI), 0.8983 rescaled consistency index $(\mathrm{RC})$ and 0.9743 retention index (RI). Whereas, the maximum parsimony tree of the ITS region was based on 45 parsimony-informative characters (PIC), with tree length, 95. The calculated matrices of this analysis were 0.1895 homoplasmy index (HI), 0.8105 consistency index (CI), 0.7286 rescaled consistency index (RC) and 0.8989 retention index (RI). These phylogenetic trees were constructed under the Bootstrap 50\% majority rule consensus (MRC) tree. These single locus MRC phylogenies depicted our fungal isolates as Independent Evolutionary

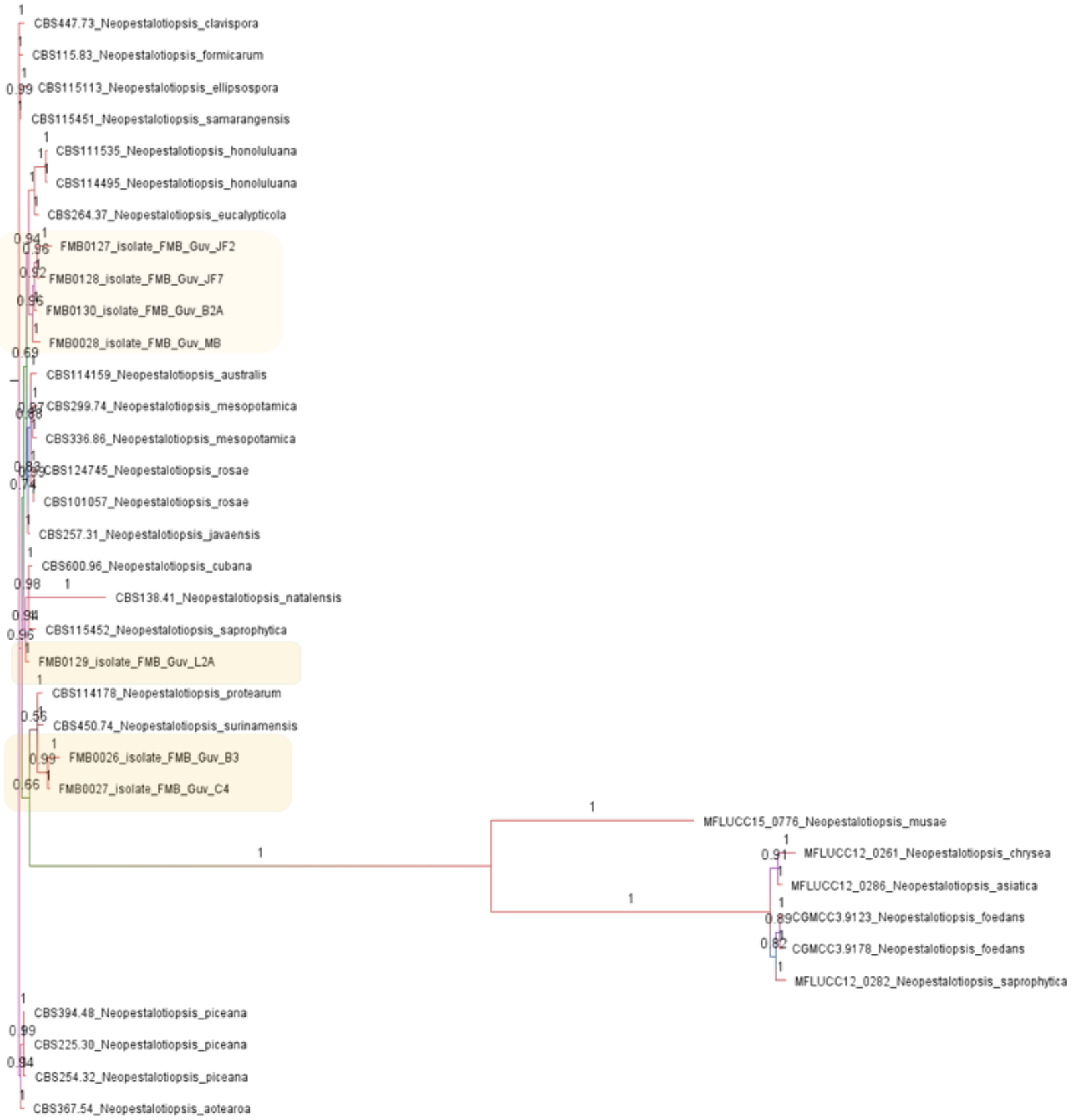

0.00

Figure 2. A phylogram of Bayesian inference (BI) analysis using a combined dataset (ITS, TEF1- $\alpha$, TUB) with MrBayes software. Scale bar is depicting the expected number of changes per site. 
Lineages (IEL). In all single-locus genealogies, these isolates were distinct and diverse compared to other species as singlelocus trees may not depict an individual as a whole to delimit species boundaries. Therefore, the concatenated analysis was performed using multiple loci-based combined dataset for genealogical concordance phylogenetic species recognition (GCPSR). In this study, this approach was based on three genes (ITS, TEF1- $\alpha$, TUB) to determine genealogies and phylogenetic topography for species recognition. The partition homogeneity test performed on the dataset of these genes with value, $\mathrm{P}=0.01$ (Cunningham, 1997; Van et al., 2007) shown that these three genes could be combined for concatenated analyses. Maximum parsimony analysis and BI were implemented on the concatenated dataset. The maximum parsimonious tree was generated through PAUP* software. The majority rule $(50 \%)$ consensus tree was based on 527 PIC with tree length, 1061. The calculated matrices of this analysis were $0.1810 \mathrm{HI}, 0.8190 \mathrm{CI}, 0.7493 \mathrm{RC}$ and 0.9148 RI. The maximum parsimony tree generated using a combined dataset of three genes (ITS, TEF1- $\alpha$, TUB) showed our fungal isolates as IELs. These isolates were not nested in clades of other species in comparison. Fungal isolates, FMB0127, FMB0128 and FMB0130, were nested in a clade while FMB0026 and FMB0027 were shown as IELs with a bootstrap value, 94\%. However, FMB0028 and FMB0129 did not form any grouping and cladding to any other members in comparison. For Bayesian analysis of the concatenated dataset, the best-fit evolutionary model $(\mathrm{SYM}+\mathrm{G})$ based on the Akaike information criterion (AIC) was selected. Markov Chain Monte Carlo was run for 5,000,000 generations and it was stopped at the average standard deviation of split frequencies, 0.0043 . The phylogenetic tree obtained through Bayesian analysis strongly support the maximum parsimony analysis generated tree. Phylogenies generated in BI were similar to MRC phylogenies obtained in MP analysis. They showed that all FMB fungal isolates were diverse and distant from other Neopestalotiopsis species. Our fungal isolates FMB0026 and FMB0027 were shown as closely related. Similarly, isolates FMB0127, FMB0128 were shown as sister isolates. Therefore, they could be different species and even different strains of a single species (Fig. 2).

Coalescent based species tree analysis for species delimitation: For generating species tree, a coalescent theorybased approach was used. This approach is different from concatenated analysis and is considered the most accurate analysis for species delimitation compared to concatenated dataset-based approaches. Therefore, Bayesian Evolutionary Analysis for Sampling Tree (BEAST) analysis was performed

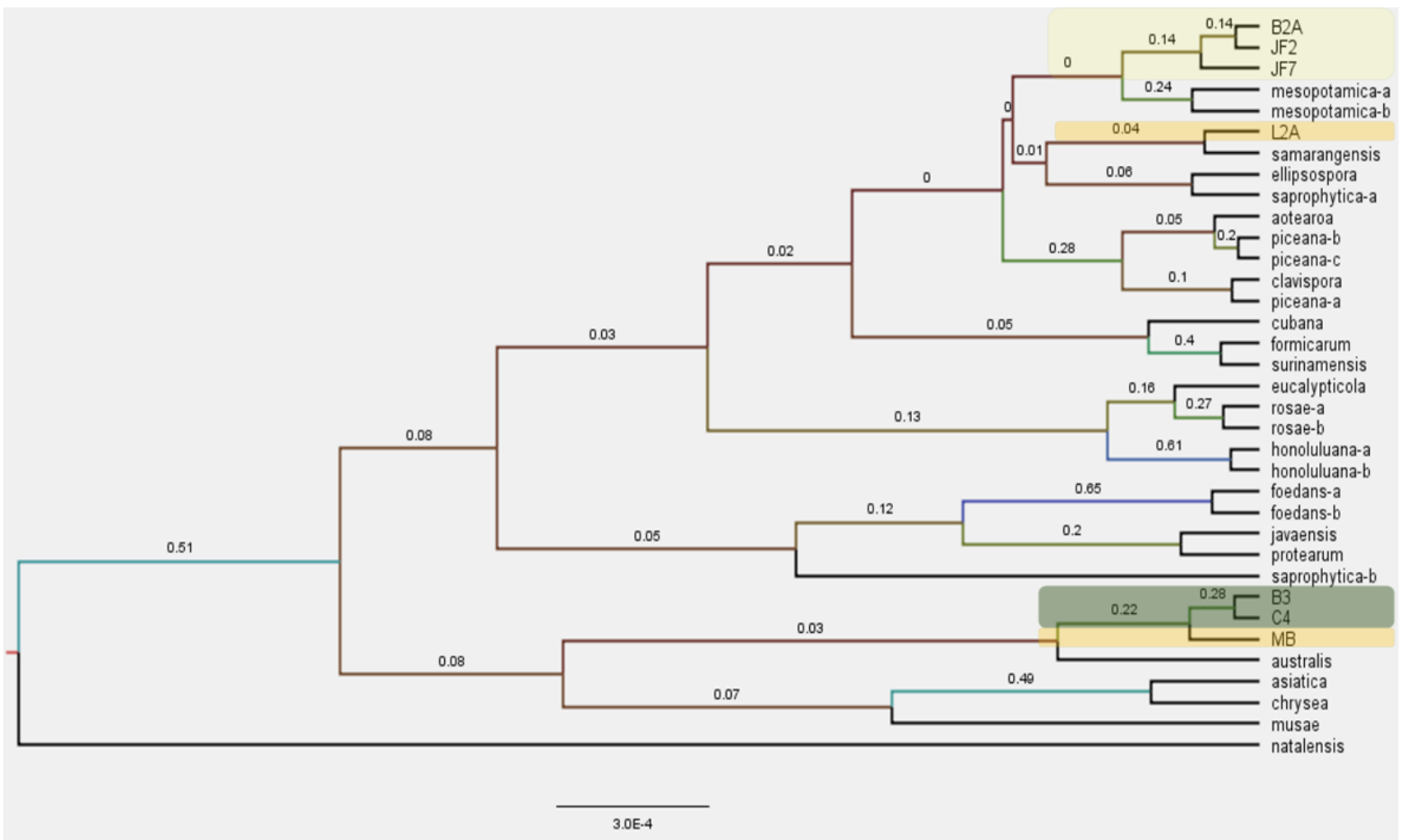

Figure 3. Species tree constructed through BEAST analysis depicted that FMB isolates are different and diverse than other operational taxonomic units (OTUs). Hence, they are proved as new Neopestalotiopsis genus species from Pakistan. These FMB fungal isolates are not just as different morphospecies, but they are also different phylogenetic species. Scale bar is depicting the expected number of changes per site. 
using *BEAST software for species tree construction. However, the species tree was visualized in Figtree software. This species tree strongly supports Bayesian inference and maximum parsimony-based phylogenies. All FMB isolates were separately grouped. This species tree demonstrated that FMB-Guv-JF2, FMB-Guv-JF7, FMB-Guv-L2A and FMBGuv-B2A were nested together. However, FMB-Guv-JF2 and FMB-Guv-JF7 showed the shortest branch length compared to other operational taxonomic units (OTUs). This revealed less evolutionary changes between these isolates. This species tree supported that these isolates are different strains of the same species. Similarly, fungal isolates FMB-Guv-B3, FMBGuv-C4 and FMB-Guv-MB made separate clade (Fig. 3). They resided in species trees as a paraphyletic group was shown distantly related to each other. This tree depicted that these FMB isolates are genetically diverse to other OTUs of this tree. They all are new species of the Neopestalotiopsis genus, which are affecting guava trees in Pakistan. Based on morphology and phylogenetic analysis, these isolates were proved as four different and new species of the genus, Neopestalotiopsis.

\section{DISCUSSION}

In Pestalotioid fungi of the Amphisphaeriaceae family, conidium's morphology is a key taxonomic character primarily used in these groups of fungi (Nag Raj, 1993; Maharachchikumbura et al., 2014). Mahachchikumbura et al. (2014) identified and introduced Neopestalotiopsis as a separate genus from Pestalotiopsis based on phylogenetic and morphological differences. However, morphological characters can be varied depending upon various factors like environmental conditions, host, etc. Maharachchikumbura et al. (2014) disagreed with the Pestalotiopsis concept for conidial forms established by Nag Raj 1993 and described 4celled conidial forms supported by phylogenetic analyses as phenotypic characteristic for all species of Pestalotiopsis, Neopestalotiopsis and Pseudopestalotiopsis genera. So, morphological segregation of Pestalotioid fungi into genera must have concordance to phylogenetic information based on gene sequence data. Hence, for phylogenetic-based welldefined generic placement of our seven fungal isolates studied (FMB isolates), generated LSU sequences were aligned with LSU sequences of Pestalotiopsis, Neopestalotiopsis and Pseudopestalotiopsis species from the database for phylogenetic analysis. Phylogenetic tree constructed by employing neighbor-joining analysis supported our morphometric characterization of these isolates as Neopestalotiopsis species. Identification of Neopestalotiopsis species at a meaningful taxonomic level (especially the identification of new species) required to couple the phenotypic analyses with phylogenetic analyses. Though morphological characterization (versicolourous median cells, indistinct conidiophores reduced to conidiogenous cells) easily distinguish Neopestalotiopsis species. In the present study, for a better understanding of the Neopestalotiopsis genus and delimiting new species in this group and morphological characters, analyses of multi-locus sequences data were also considered. Because overlapping phenotypic characteristics usually confuses the taxonomists and makes the segregation of morphologically similar taxa more difficult. Jeewon et al., 2003 made the first comprehensive phylogenetic study based on ITS sequence data in Pestalotiopsis for evaluating the phylogenetic importance of its morphological attributes in taxonomy. However, Hu et al. (2007) put forwarded TUB gene region's significance to resolve phylogenetic boundaries in Pestalotioid fungi. Maharachchikumbura et al. (2012) proved ITS, TUB and TEF regions as comparatively better molecular markers out of 10 tested gene regions, including actin, ITS, LSU, TUB, TEF, Calmodulin, glyceraldehyde-3-phosphate dehydrogenase, glutamine synthase, RNA polymerase II and $18 \mathrm{~S}$ nrDNA to resolve species delimitation at satisfactory level. They introduced 11 new species in the Neopestalotiopsis genus by investigating ITS, TUB and TEF regions combined with morphological data (Maharachchikumbura et al., 2014). Therefore, in this study, we used the phylogenetic investigation of our seven fungal isolates using ITS, TEF1- $\alpha$, and TUB gene regions. These genic regions were analyzed for phylogenetic species recognition by subjecting their data partition and their concatenated dataset to different phylogenetic analysis methods based on different algorithms and assumptions. For delimiting species boundaries of studied Neopestalotiopsis isolates, results of single-locus-based phylogenetic trees were supplemented by phylogenetic trees generated with the multilocus-based concatenated dataset and species tree generated with the coalescent theory-based approach to validate species identification. All phylogenetic trees resolved these FMB isolates of Neopestalotiopsis as distantly related isolates of diverse genetic backgrounds to other operational taxonomic units in comparison. Morphometric characterization of these isolates proved them as four different morphospecies of the Neopestalotiopsis genus. Based on phylogenetic trees (generated using single-locus dataset and multiple loci-based concatenated dataset analyses) and species tree (generated using coalescent theory-based analysis), they also proved four different phylogenetic species of Neopestalotiopsis genus. Hence, by fulfilling species criteria using morphological species concept and phylogenetic species concept, these seven FMB isolates were showed as divergent evolutionary lineages and identified as four new Neopestalotiopsis genus species, named as Neopestalotiopsis guajavae I.U Haq, S. Ijaz \& N.A. Khan, sp.nov.; Neopestalotiopsis psidii I.U Haq, S. Ijaz \& N.A. Khan, sp.nov.; Neopestalotiopsis perukae I.U Haq, S. Ijaz \& N.A. Khan, sp.nov.; Neopestalotiopsis guajavicola I.U Haq, S. Ijaz \& N.A. Khan, sp.nov. 
Conclusion: Neopestalotiopsis as a separate genus from Pestalotiopsis based on phylogenetic and morphological differences has been declared. However, overlapping this group's phenotypic characteristics usually confuses the taxonomists and makes the segregation of morphologically similar taxa more difficult. Therefore, in the present study, for a better understanding of the Neopestalotiopsis genus and delimiting new species in this group and morphological characters, analyses of multi-locus sequences data are also considered. Hence, by fulfilling species criteria using morphological species concept and phylogenetic species concept, seven FMB isolates showed divergent evolutionary lineages and identified four new species of the Neopestalotiopsis genus.

\section{REFERENCES}

Abbas, S.Q., M. Niaz, T. Iftakhar, A. Perveen, A. Abbas and R. Sana. 2014. New Fungal Records on Guava (Psidium guajava) from Pakistan. PAS. 51:121-127.

Amusa, N.A., O.A. Ashaye, M.O. Oladapo and M.O. Oni. 2005.Guava fruit anthracnose and the effects on its nutritional and market values in Ibadan, Nigeria. World J. Agric. Res. 1:169-172.

Carbone, I. and L.M. Kohn. 1999. A method for designing primer sets for speciation studies in filamentous ascomycetes. Mycologia. 91:553-556.

Castresana, J. 2000. Selection of conserved blocks from multiple alignments for their use in phylogenetic analysis. Mol. Biol. Evol.17:540-552.

Cunningham, C.W. 1997. Can three incongruence tests predict when data should be combined? Mol. Biol. Evol. 14:733-740.

Denman, S., W.C. Pedro, E.T. Joanne, K. Ji-Chuan, P. Ian and J.W. Michael. 2000. An overview of the taxonomic history of Botryosphaeria and a re-evaluation of its anamorphs based on morphology and ITS rDNA phylogeny. Stud. Mycol. 45:129-140.

Dettman, J.R., J.J. David and W.T. John. 2003. A multi-locus genealogical approach to phylogenetic species recognition in the model eukaryote Neurospora. Evolution. 57:2703-2720.

Fong, Y.K., S. Anuar, H.P. Lim, F.Y. Tham and F.R. Sanderson. 2000. A modified filter paper technique for long-term preservation of some fungal cultures. Mycologist. 14:127-130.

Glass, N.L. and G.C. Donaldson. 1995. Development of primer sets designed for use with the PCR to amplify conserved genes from filamentous ascomycetes. Appl. Environ. Microbiol. 61:1323-1330.

Guava production in 2018. Tridge. 2019. Retrieved 16 November 2020.

Guba, E.F. 1961. Monograph of Pestalotia and Monochaetia. Cambridge: Harvard University Press.
Hu, H., R. Jeewan, D. Zhou, T. Zhou and K.D. Hyde. 2007. Phylogenetic diversity of endophytic Pestalotiopsis species in Pinus armandii and Ribes spp.: evidence from rDNA and $\beta$-tubulin gene phylogenies. Fungal Divers. 24:1-22.

Hyde, K.D., R.H. Nilsson and S.A. Alias. 2014. One stop shop: backbones trees for important phytopathogenic genera: I. Fungal Divers. 67:21-125.

Jayawardene, R.S., M. Liu, S.S.N. Maharachchikumbura. 2016. Neopestalotiopsis vitis sp. nov. causing grapevine leaf spot in China. Phytotaxa. 258: 63--74.

Jeewon, R., E.C.Y. Liew and J.A. Simpson. 2003. Phylogenetic significance of morphological characters in the taxonomy of Pestalotiopsis species. Mol. Phylogenet. Evol. 27:372-383

Kohn, L.M. 2005. Mechanisms of fungal speciation. Annu. Rev. Phytopathol. 43:279-308

Maharachchikumbura, S.S.N., E. Chukeatirote and L.D. Guo. 2013. Pestalotiopsis species associated with Camellia sinensis (tea). Mycotaxon. 123:47-61.

Maharachchikumbura, S.S.N., L.D. Guo and L. Cai. 2012. A multi-locus backbone tree for Pestalotiopsis, with a polyphasic characterization of 14 new species. Fungal Divers. 56:95-129.

Maharachchikumbura, S.S.N., D.H. Kevin, Z.G. Johannes, J. $\mathrm{Xu}$ and W.C. Pedro. 2014. Pestalotiopsis revisited. Stud. Mycol. 79:121-186.

Misra, A.K. 2004. Guava diseases - their symptoms, causes and management. In: Naqvi SAMH. Diseases of Fruits and Vegetables. Volume II. Dordrecht: Springer. p. 81119.

Nag Raj, T.R. 1993. Coelomycetous anamorphs with appendage-bearing conidia. Mycologue Publications.

O'Donnell, K. and E. Cigelnik. 1997. Two divergent intragenomic rDNA ITS2 types within a monophyletic lineage of the fungus Fusarium are nonorthologous. Mol. Phylogenet. Evol. 7:103-116.

O'Donnell, K., H.C. Kistler, E. Cigelnik and R.C. Ploetz. 1998. Multiple evolutionary origins of the fungus causing Panama disease of banana: concordant evidence from nuclear and mitochondrial gene genealogies. Proc. Natl. Acad. Sci. 95:2044-2049.

Pavlic, D., B. Slippers, T.A. Coutinho and M.J. Wingfield. 2007. Botryosphaeriaceae occurring on native Syzygium cordatum in South Africa and their potential threat to Eucalyptus. Plant Pathol. 56:624-636.

Pervaiz, U., A. Khan, R. Javed and J. Zeb. 2008. Production constraints of guava in district Kohat. Sarhad J. Agric. 24:549-554.

Rintoul, T.L., A.E. Quinn and L.C. André. 2012. Multigene phylogenetic analyses to delimit new species in fungal plant pathogens. Plant Fungal Pathogens. pp. 549-569.

Smith, J.A. and P.E. Todd. 2001. Reconciling conflicting evidence on the performance of propensity-score 
matching methods. American Economic Review. 91:112-8

Sutton, B.C. 1980. The Coelomycetes, Fungi Imperfecti with Pycnidia. acervuli and stromata. Kew: Commonwealth Mycological Institute.

Taylor, J.W., D.J. Jacobson, S. Kroken, T. Kasuga, D.M. Geiser, D.S. Hibbett and M.C. Fisher. 2000. Phylogenetic species rec-ognition and species concepts in fungi. Fungal Genet. Biol. 31:21-32.

Van Wyk, M., A.O. Al Adawi, I.A. Khan, M.L. Deadman, A.A. Al Jahwari, B.D. Wingfield, R. Ploetz and M.J. Wingfield. 2007. Ceratocystis manginecans sp. nov., causal agent of a destructive mango wilt disease in Oman and Pakistan. Fungal Divers. 27:213-230.

Vilgalys, R. and M. Hester. 1990. Rapid genetic identification and mapping of enzymatically amplified ribosomal DNA from several Cryptococcus species. J. Bacteriol. 172:4238-4246.

White, T.J., T. Bruns, S.J.W.T. Lee and J. Taylor. 1990. Amplification and direct sequencing of fungal ribosomal RNA genes for phylogenetics. In: M.A. Innis, D.H. Gelfand, J.J. Sninsky and T.J. White. PCR protocols: a guide to methods and applications(ed). San Diego: Academic Press.315-322. 